\title{
Changes in tension regulates proliferation and migration of fibroblasts by remodeling expression of ECM proteins
}

\author{
MINMIN JIANG ${ }^{1,2}$, JUHUI QIU ${ }^{1,3}$, LINGLING ZHANG ${ }^{1,3}$, DONGYUAN LÜ ${ }^{4}$, \\ MIAN LONG ${ }^{4}$, LI CHEN ${ }^{1,3,5}$ and XIANGDONG LUO ${ }^{1,3}$ \\ ${ }^{1}$ Burn Research Institute, Southwest Hospital, Third Military Medical University, Chongqing 400038; \\ ${ }^{2}$ Central Laboratory, The People's Hospital of Guizhou Province, Guiyang, Guizhou 550002; \\ ${ }^{3}$ National Key Laboratory of Trauma and Burns and Chongqing Key Laboratory of Disease Proteomics; \\ ${ }^{4}$ Key Laboratory of Microgravity (National Microgravity Laboratory) and Center for Biomechanics and Bioengineering, \\ Institute of Mechanics, Chinese Academy of Sciences, Beijing $100190 ;{ }^{5}$ Breast Disease Center, \\ Southwest Hospital, Third Military Medical University, Chongqing 400038, P.R. China
}

Received June 4, 2015; Accepted May 18, 2016

DOI: $10.3892 /$ etm.2016.3497

\begin{abstract}
Wound healing is a complicated but highly organized process in which cell migration and proliferation are actively involved. However, the process by which mechanical stretch regulates the proliferation and migration of human skin fibroblasts (HFs) and keratinocytes is poorly understood. Using a house built mechanical stretch device, we examined the HFs extracellular matrix (ECM) components changes under non-stretch, static stretch or cyclic stretch conditions. We further investigated the changes in ECM component protein expression levels in keratinocytes and analyzed the effects of individual ECM component on keratinocyte proliferation and migration. Particularly, the roles of calcium/calmodulin-dependent serine protein kinase (CASK) in the HF proliferation under cyclic stretch were investigated. Cyclic stretch suppressed HF proliferation compared with HFs without stretch or with static stretch. Cyclic stretch also led to a significant reduction in the levels of collagen I and a marked increase of fibronectin in HFs ECM. By contrast, collagen I levels increased and fibronectin levels decreased in response to non-stretch and static stretch conditions. After cyclic stretch, the proliferation of keratinocytes was inhibited by the cyclic
\end{abstract}

Correspondence to: Dr Li Chen, Breast Disease Center, Southwest Hospital, Third Military Medical University, 30 Gaotanyan Street, Chongqing 400038, P.R. China

E-mail: lichen201501@sian.com

Dr Xiangdong Luo, Burn Research Institute, Southwest Hospital, Third Military Medical University, 30 Gaotanyan Street, Chongqing 400038, P.R. China

E-mail: xiangdongluo2015@sina.com

Key words: human skin fibroblast, keratinocyte, mechanical stretch, extracellular matrix, integrin, calcium/calmodulin-dependent serine protein kinase stretch-induced ECM in HFs. The inoculation of keratinocytes with single ECM component suggested that collagen I was more capable of inducing cell proliferation than fibronectin, while it had less impact on cell migration compared with fibronectin. Furthermore, cyclic stretch induced by proliferation inhibition was associated with altered integrin $\beta 1$-CASK signal pathway. The present results demonstrated the existence of HF-ECM-keratinocyte 'cross-talk' in cutaneous tissues. Thus, the integrin $\beta 1-\mathrm{CASK}$ signal pathway in HFs may be involved in the outside-in signal transduction of extracellular stretch and the altered ECM component expression.

\section{Introduction}

The existence of skin tension has been reported for $\sim 200$ years (1). As a result of muscle activity and respiration, the majority of the skin covering a body is in the status of cyclic tension. Although the pathophysiological roles of skin tension in certain diseases have been initially investigated (2), the biological significance of tension signal transduction in skin cells are poorly understood, particularly the roles of tension in the healing of skin wound (3). Normally, cutaneous tissues and cells, particularly the dermal tissue and fibroblasts, are the major tissues and cellular type to receive signals and respond towards tension (1). Although the keratinocytes in the epidermis endure tension weakly, they receive the signals from cytokines and extra cellular matrix (ECM) secreted by human skin fibroblasts (HFs) in dermal tissue $(1,4)$. Once skin tension disappears due to skin injury, multiple cellular processes will be induced in cutaneous tissues to accelerate wound healing (4). At the molecular level, the sudden disappearance of skin tension may induce the expression of various proteases in keratinocytes, which degrade particular proteins of ECM to facilitate the migration and re-epithelization of keratinocytes (5-8).

The maintenance of cell normal function relies substantially on the ECM (9). During the process of cell signal transduction, the receptors in cell membrane bind to specific 
ECM components to form the 'cross-talk' between cells and ECM. The interaction further mediates transmembrane signal transduction (10,11). The interaction between cells and ECM assists in maintaining normal cellular physiological activities, in addition to playing an important function in multiple pathological processes, including wound healing, scar formation and tissue fibrosis (10). Therefore, it has been widely accepted that cell-ECM mediated transmembrane signal transduction is a major pathway for skin to receive the outside mechanical tension signal (10).

During the initial process of skin injury, mechanical tension vanishes in the edge of the wound, numerous temporary matrices are presented in the surface of wound, and inflammatory cell infiltration is followed by neovascularization and the aggregation of HFs (12). In the post stage of wound healing, the surface of wound shrinks, and the temporary matrices in the surface are substituted by mature matrices generated from aggregated HFs, and finally scars are formed (12). Nevertheless, it remains unclear how the mechanical tension signal is transduced from HFs to keratinoctyes in the process of sudden skin tension disappearance (10).

The calcium/calmodulin-dependent serine protein kinase (CASK), a membrane-associated guanylate kinase and a scaffolding protein, has been demonstrated to recruit or organize other proteins at the plasma membrane to coordinate signal transduction pathways within the cytoplasm and nucleus (13). Sun et al (14) reported that CASK regulates the protein and mRNA level of p21, which promotes cell proliferation in ECV304 cells. Furthermore, CASK has been indicated to participate in the control of the G0-G1 restriction check point of the cell cycle in human breast cancer cells via inhibiting cyclin D1 synthesis and pRb phosphorylation (15). Thus, CASK serves a crucial function in cell growth, while little is known about CASK effect under cyclic stretch.

In the present study, we hypothesize that the 'cross-talk' among HFs, ECM (HF-secreted) and keratinocytes exists in cutaneous tissues. In such cell-ECM-cell interaction processes, HFs may modulate the expression levels of specific ECM proteins in response to skin tension changes. The ECM component change would subsequently affect the proliferation and migration of keratinocytes. To test this hypothesis, we employed a in-house built mechanical stretch device to apply mechanical force against the cultured HF cells. By applying three different mechanical stretches, including non-stretch, static stretch or cyclic stretch, we quantified the expression levels change of ECM proteins in and investigated the molecular signaling transduction pathways in the process of mechanical tension-induced HF proliferation. We further examined the effects of ECM protein expression changes on the proliferation and migration of keratinocytes. CASK has been demonstrated to involve in cell growth and calcium signaling, so we next investigated the effect of CASK on HF proliferation under cyclic stretch.

\section{Materials and methods}

Antibody and reagents. Mouse monoclonal antibodies against human collagen I, III, IV, fibronectin, CASK and integrin $\beta 1$ were purchased from Santa Cruz Biotechnology, Inc. (Santa
Cruz, CA, USA). Collagen I, fibronectin and all other reagents were purchased from Sigma-Aldrich (St. Louis, MO, USA).

Cells and cell culture. Human skin fibroblasts [CRL2088; American Type Culture Collection (ATCC), Manassas, VA, USA] and spontaneously immortalized keratinocyte HaCaT (CRL2309; ATCC) were used in this study. HaCaT cells were maintained in RPMI-1640 medium (HyClone; GE Healthcare, Logan, UT, USA) containing 10\% fetal bovine serum (FBS; Gibco; Thermo Fisher Scientific, Inc., Grand Island, NY, USA) and $1 \%$ penicillin/streptomycin (HyClone; GE Healthcare). HFs were cultured in Dulbecco's modified Eagle's medium (1 g/l glucose) with $10 \%$ FBS and $1 \%$ antibiotics. When reaching $\sim 80 \%$ confluence, cells were harvested and then transferred to the mechanical stretch device developed in-house for an additional 6 days of culture under mechanical stretch (Fig. 1A). Cells were then harvested at 0, 1, 2, 3, 4 and 5 days for various functional investigations.

Application of cyclic stretch to cultured cells. Mechanical stretch was applied to examine mechano-transduction of HFs. HF cells grown in a flask were transferred onto the silicone membrane pre-treated with oxygenized plasma. A non-toxic stainless steel frame was used to retain the cells inside the seeding region. The membrane was then mounted to the mechanical stretch device and experienced a cyclic stretch with $20 \%$ strain and $1 \mathrm{~Hz}$ frequency for 6 days. The physiological stretch frequency is from 0.1 (respiratory rate) to $1.25 \mathrm{~Hz}$ (heart rate), all frequency in this arranges can be used for cyclic stretch. In this study $1 \mathrm{~Hz}$ was used in our pre-experiments, as previous reports have suggested that $1 \mathrm{~Hz}$ frequency is appropriate for cyclic stretch investigation $(16,17)$. Cells under static stretch with $20 \%$ strain and non-stretch case were used as a control.

ECM preparation and extraction. For ECM preparation, HF cells under different stretches were maintained for up to 6 days. Cells were then washed with sterile distilled water for $4 \mathrm{~h}$ followed by $1 \mathrm{mM} \mathrm{NaCl}$ for $1 \mathrm{~h}$. A total of three times of wash cycles were applied to cells. Finally, the cells were dried overnight at $4^{\circ} \mathrm{C}$. HaCaT cells were then seeded on the prepared ECM on silicone membrane. For ECM extraction, remaining ECM on silicone membrane was lysed with RIPA buffer containing protease inhibitors and complete mini EDTA-free (Roche Diagnostics, Branchburg, NJ, USA) for western blot.

ECM preparation. A certain quantity of liquid silicone was solidified in 24-well plates to form a silicone membrane, on which $10 \mu \mathrm{g} / \mathrm{ml}$ collagen I or human fibronectin (BD Biosciences, San Jose, CA, USA) was coated for at $37^{\circ} \mathrm{C}$. The silicone membrane was then washed thoroughly with PBS and seeded with $1 \times 10^{4}-2 \times 10^{4} \mathrm{HaCaT}$ cells.

Western blot and immunoprecipitation. Cells were pelleted and lysed directly using 2X Laemmli sample buffer (1610737, Bio-Rad Laboratories, Inc., Hercules, CA, USA) with BME. The cell lysate was then incubated at $95^{\circ} \mathrm{C}$ for 5 mins and stored at $-20^{\circ} \mathrm{C}$ until the samples were run on an SDS-PAGE gel. Protein loading was quantified using the Pierce BCA 
Protein Assay kit (23225; Thermo Fisher Scientific, Inc.). Western blot analysis was conducted according to previous reports $(18,19)$. Samples of crude proteins $(20 \mu \mathrm{g})$ were fractionized using 4-20\%SDS-PAGE Tris-glycine gels (Thermo Fisher Scientific, Inc., Waltham, MA, USA) and transferred onto nitrocellulose membranes. Membranes were blocked for $1 \mathrm{~h}$ with $5 \%$ milk in Tris-buffered saline, then incubated with the following primary monoclonal antibodies in 5\% milk with $0.1 \%$ Tween 20 for $16 \mathrm{~h}$ at $4^{\circ} \mathrm{C}$ : Collagen I (cat. no. ab34710: Abcam, Cambridge, UK), Collagen III (cat. no. ab7778; Abcam), Collagen IV (cat. no. ab6586; Abcam), fibronectin (cat. no. ab3413; Abcam), integrin $\beta 1$ (cat. no. ab179472; Abcam), CASK (cat. no. ab99039; Abcam), integrin $\beta 3$ (cat. no. ab119992; Abcam), $\beta$-actin (cat. no. 4970; Cell Signaling Technology, Inc., Dancers, MA, USA), EGF (cat. no. 2646; Cell Signaling Technology, Inc.), FGF (cat. no. 9740; Cell Signaling Technology, Inc.), PDGF (cat. no. 3169; Cell Signaling Technology, Inc.) and IGF (cat. no. 9750; Cell Signaling Technology, Inc.). All antibodies were diluted 1:1,000. After washing with Tris-buffered saline Tween 20 the membranes were then incubated for $1 \mathrm{~h}$ at $4^{\circ} \mathrm{C}$ with a goat anti-mouse secondary antibody conjugated with horseradish peroxidase (1:5,000; 31430; Thermo Fisher Scientific, Inc.). After washing, membranes were incubated for 1 min with Western Lightning Chemiluminescence reagent Plus (PerkinElmer, Inc., Waltham, MA, USA) and the signal of western blot was exposed and developed. Band intensities were visualized using Pierce ECL Western blotting (32106; Thermo Fischer Scientific, Inc.) and quantified using Image $\mathbf{J}$ (National Institutes of Health, Bethesda, MD, USA). Immunoprecipitation was performed as previously reported (16). Briefly, HFs were lysed in ice-cold RIPA buffer containing protease inhibitors. $400 \mu \mathrm{g}$ cell lysate proteins was then incubated with $14 \mu 1$ mouse anti-human CASK (1:1,000; ab99039; Abcam) or anti-human integrin $\beta 1$ (1:1,000; ab179472; Abcam) monoclonal antibody for $2 \mathrm{~h}$ at $4^{\circ} \mathrm{C}$. Next, $20 \mu \mathrm{l}$ protein $\mathrm{G}$-agarose was added afterwards and incubated at $4^{\circ} \mathrm{C}$ overnight on a rocker platform. Following centrifugation at $1,000 \mathrm{x}$ for $5 \mathrm{~min}$ at $4^{\circ} \mathrm{C}$, the agarose beads were washed four times with RIPA buffer at $4^{\circ} \mathrm{C}$ and lysed in the sample buffer for further western blot analysis as described above. Antigen retrieval was performed using citrate buffer [10 mM citric acid, $0.05 \%$ Tween $20,(\mathrm{pH} \mathrm{6.0)}]$ at $95-100^{\circ} \mathrm{C}$ using a pre-heat steamer.

Mammalian two-hybrid protein-protein interaction assays. This experiment was performed as described previously (20). Briefly, the entire coding sequence of human CASK was cloned in-frame into a pM vector (Clontech Laboratories, Inc., Mountain View, CA, USA) encoding the GAL4 DNA binding domain. The cDNAs of integrin $\beta 1$ extracellular or intracellular terminal were donated by Dr Xinyu Wang of the Shanghai Institute of Biochemistry and Cell Biology (Chinese Academy of Sciences, Shanghai, China). These were cloned respectively in-frame into the pVP16 vector (Clontech Laboratories, Inc.) encoding the VP16 transactivation domain. The pG5CAT reporter vectors $(0.5 \mu \mathrm{g})$ containing a chloramphenicol acetyltransferase (CAT) reporter gene under the control of the GAL4 response element and $2.5 \mu \mathrm{g}$ of each of the above-mentioned constructed vectors were co-transfected into $2.5 \times 10^{5} \mathrm{HFs}$ per well in six-well plates with the LF2000 reagent (Gibco;
Thermo Fisher Scientific, Inc.). After $48 \mathrm{~h}$ of transfection, cells were harvested and extracts were assayed for CAT activity using a CAT ELISA assay kit (Roche Diagnostics).

Analysis of cell proliferation and migration. Cells were seeded onto silicone membranes in 24-well plates at a density of $1-2 \times 10^{4}$ cells per well. At each time-point, cells were harvested after trypsinization and centrifugation at $800 \mathrm{x} \mathrm{g}$ for $5 \mathrm{~min}$ at $4^{\circ} \mathrm{C}$. All samples were prepared in quadruplicate and the entire experiment was repeated twice. Cells were plated onto silicone membrane pre-coated with collagen I or fibronectin followed by culture for $\sim 24 \mathrm{~h}$. When the cell growth reached $80 \%$ confluence with monolayer, one $200 \mu 1$ pipette tip was used to straightly scratch cells to create wound. Between two and four scratches were made in each well. Cells were photographed using an inverted microscope (Olympus Corporation, Tokyo, Japan) at day 0, 1, 2, 3, 4 and 5, and the wound area was measured using UTHSCSA image tool software version 3.0 (University of Texas Health Science Center, San Antonio, TX, USA). The migration rate (MR) was calculated using the formula: $\mathrm{MR}(\%)=(1-\mathrm{At} / \mathrm{A} 0) \mathrm{x} \%(\mathrm{~A} 0$ : area at $0 \mathrm{~h}$; At: area at indicated times).

Cell cycle analysis. Cells were harvested at the indicated time points and stained with propidium iodide using Cycletest Plus (BD Biosciences, San Jose, CA, USA) according to the manufacturer's instructions. Cells were then analyzed using FACSCalibur with ModFit LT software, version 2.0 (BD Biosciences) to demonstrate G0/G1, S, G2/M and hypodiploid nuclei.

Cell transfection and RNA interference. siRNAs targeted hCASK mRNAs were designed and synthesized by Ambion (Thermo Fisher Scientific, Inc., Austin, TX, USA). A sequence of 19 nucleotides within the hCASK coding region immediately downstream of AA dinucleotides was selected as the target sequence $(5,21)$. Two oligonucleotides were synthesized, annealed, and inserted into the BamHI and HindIII digestion sites of the pSilencer 3.1-H1 hygro vector (Ambion; Thermo Fisher Scientific, Inc.), as described in our previous report $(5,21)$. HF cells were transfected with expression plasmids of CASK siRNA or empty vector pBS/U6 by FuGENE6 reagent (Roche Diagnostics) according to the manufacturer's instructions. Stable transformants were obtained under the selection with $200 \mu \mathrm{g} / \mathrm{ml}$ hygromycin B (Stratagene California, La Jolla, CA, USA). The positive stable clones were referred to as siCASK.

Statistical analysis. Data were analyszed using GraphPad Software (GraphPad Software, Inc., LaJolla, CA, USA) and presented as the means \pm standard error of the mean. Student's unpaired t-tests (two-tailed) were used to determine the statistical differences between groups. $\mathrm{P}<0.05$ was considered to indicate a statistically significant difference.

\section{Results}

Cyclic stretch inhibited HF proliferation. We initially employed our in-house built cell stretch device to apply a cyclic or static stretch on HF seeded in the central region of silicone 
A

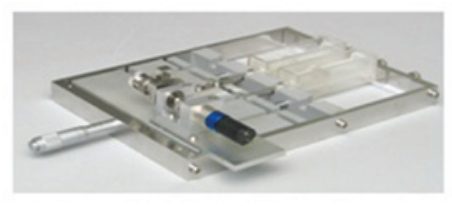

D

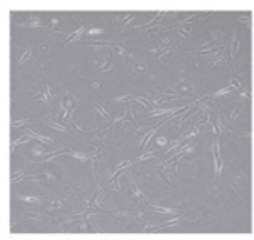

Non Stretch

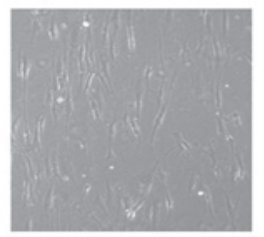

Cyclical Stretch
B

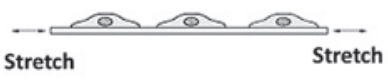

C

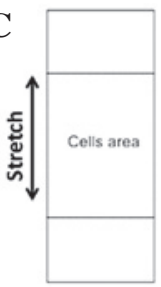

E

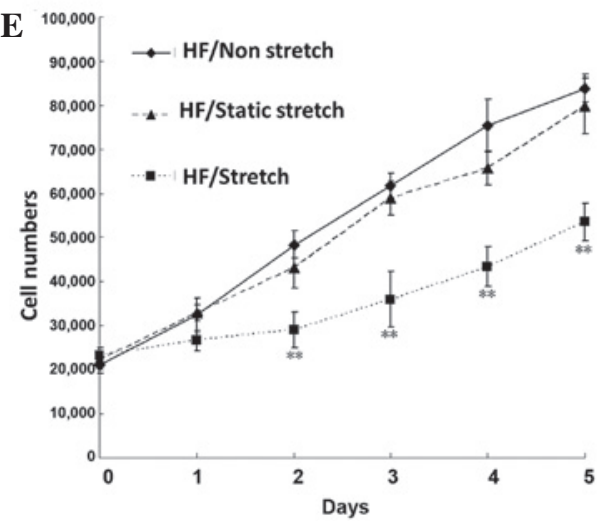

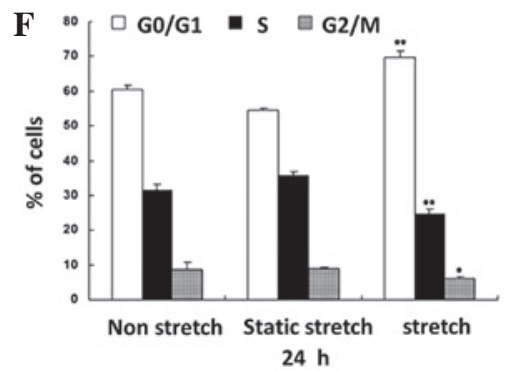

$24 \mathrm{~h}$

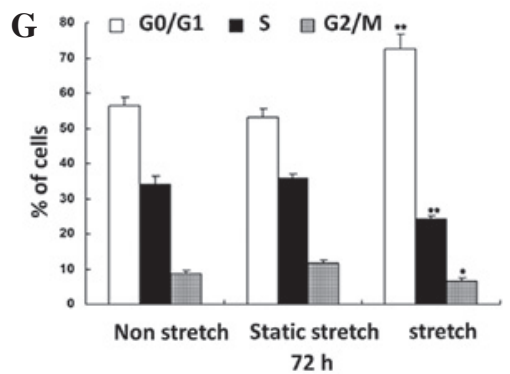

$72 \mathrm{~h}$

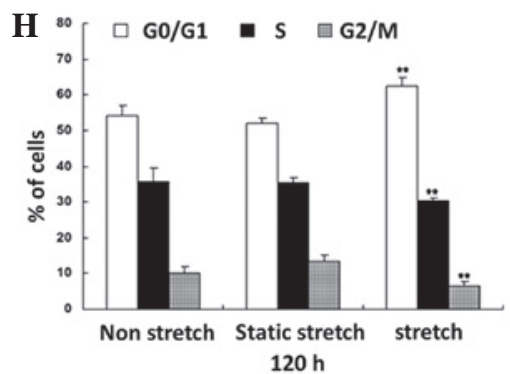

$120 \mathrm{~h}$

Figure 1. Cyclic stretch induces the morphologic changes of HFs and inhibits HFs proliferation. (A) Set up of the in-house built cell mechanical stretch device. (B) A typical pattern of stretch using $20 \%$ strain via a stretched silicone membrane. (C) Scheme demonstrating the way stretch was applied to the cells seeded in the central region of the membrane. (D) Morphology of HF cells without stretch (left panel) and under cyclic stretch (right panel). (E) Total HF cell numbers after 5 days of culture under the condition of non-stretch, static stretch or stretch. Cell cycle analysis of HF cells cultured for (F) $24,(\mathrm{G}) 48$ and (H) $120 \mathrm{~h}$ under the conditions of non-stretch, static stretch or stretch. Data are presented as the mean $\pm_{-}$standard error. "P $<0.05$ vs. static stretch or without stretch group. ${ }^{* *} \mathrm{P}<0.01$ vs. static stretch or without stretch group. HF, human skin fibroblast.

gel membrane where the mechanical tension is uniform (Fig. 1A-C). In contrast to the minimal morphological change of HF cells without mechanical stretch (Fig. 1D, left panel), cells under cyclic stretch showed a significant morphological change with stretch-directed orientation perpendicular to the direction of stretch (Fig. 1D, right panel).

Cells undergone cyclic stretch also revealed a decreased degree of proliferation compared with those under static stretch or non-stretch. First, HFs cultured under cyclic stretch had a significantly decreased number of total cells after 5 days of culture compared with those were cultured under non-stretch or statical stretch (both $\mathrm{P}<0.01$ ). To further prove the decreased cell proliferation in HFs under cyclic stretch condition, we performed flow cytometry to demonstrate the cell cycle change in these HFs. We found that the percentage of G0/G1 phase HF cells after 24,72 and $120 \mathrm{~h}$ of cyclic stretch was markedly higher compared with under static stretch or non-stretch for the same length of culture (both $\mathrm{P}<0.01$; Fig. 1E). By contrast, a significantly lower percentage of cells cultured under cyclic stretch entered $\mathrm{S}$ phase compared with the other two stretch conditions (both $\mathrm{P}<0.01$; Fig. 1E).
Altogether, these data suggested that cyclic stretch inhibits HF proliferation whereas static stretch or non-stretch did not.

Cyclic stretch regulates the specific component expression of ECM of HFs. The underlying mechanism by which cyclic stretch inhibits HF proliferation remains elusive. Although previous studies suggested that the autocrine or paracrine growth factors secreted by HFs in response to stretch were involved in the process (22), our independent investigations with the co-culture of HF and keratinocytes showed that the effects of HF growth factors alone was not sufficient to explain the mechanism of proliferation inhibition (data not shown). We hypothesized that HFs could modulate the specific component expression of HF ECM in response to cyclic stretch, which subsequently affected the proliferation and migration of keratinocytes. To validate this, we extracted the ECM of HFs under cyclic stretch, static stretch and non-stretch conditions. The differences in the expression levels of major ECM components including collagen I, III, IV and fibronectin were investigated using western blot analysis. The results indicated that the expression levels of collagen III and IV 


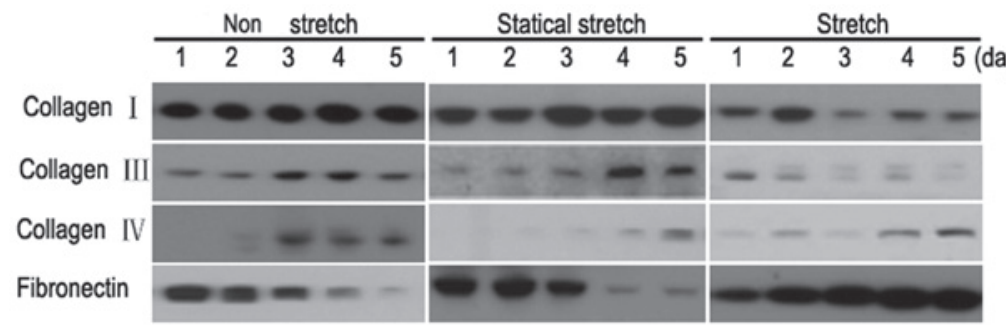

Figure 2. Cyclic stretch significantly altered the extracellular matrix component protein expression levels compared with static stretch and non-stretch, with decreased collagen III and IV, and increased fibronectin levels.
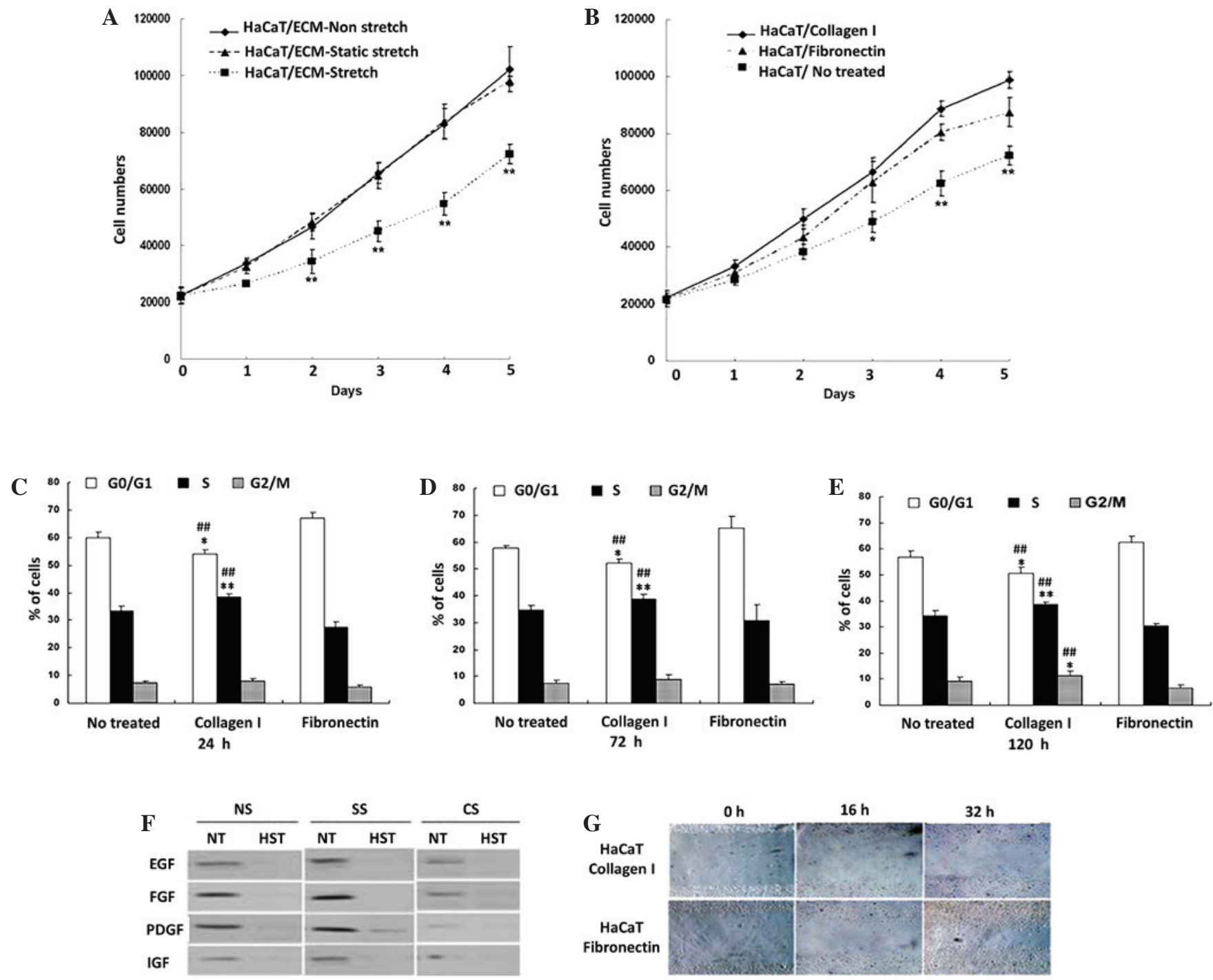

Figure 3. Cyclic stretch induced HF ECM inhibited the proliferation of keratinocytes. (A) Total HaCaT cell numbers after 5 days of culture on silicone gel membranes with the prepared ECM from HFs under cyclic stretch, static stretch or non-stretch ( $\mathrm{n}=3$ ) ${ }^{* * *} \mathrm{P}<0.01 \mathrm{vs.}$ non-stretch or static stretch group. (B) Total $\mathrm{HaCaT}$ cell numbers after 5 days of culture on silicone gel membranes coated with collagen I, fibronectin or blank matrix for indicated period of times. ${ }^{* *} \mathrm{P}<0.01$ vs. fibrinogen coated or no treated membrane. Cell cycle profiles of $\mathrm{HaCaT}$ cells cultured on silicone gel membranes with collagen I, fibronectin or blank matrix for (C) 24, (D) 72 and (E) $120 \mathrm{~h} .{ }^{* *} \mathrm{P}<0.01,{ }^{\# \#} \mathrm{P}<0.01$ vs. no treated membrane. (F) Western blot of growth factors including EGF, FGF, PDGF and IGF in extracted HF ECM upon S, SS or NS treated HF. ECM on silicone gel membranes were eluted with HST or NT. (G) Scratch migration assay of HaCaT cells on the silicone gel membrane with collagen I or fibronectin matrix. The influence of matrix proteins on cellular migration by wound-healing assay at the time of 16 and $32 \mathrm{~h}$ of post scratched respectively. ${ }^{*} \mathrm{P}<0.05$ vs static stretch or without stretch group. HF, human skin fibroblast; ECM, extracellular matrix; S, cyclic stretch; SS, static stretch; NS, non-stretch; HST, high salt solution; NT, without high salt solution.

were significantly higher in HFs cultured under cyclic stretch compared with static stretch and non-stretch (Fig. 2). By contrast, the expression of fibronectin was markedly reduced under this condition compared with those cultured with static stretch and non-stretch (Fig. 2). Furthermore, the increase in collage III or IV and decrease in fibronectin levels occurred 
in a time-dependent manner with 5 days of culture having the most significant difference (Fig. 2).

Protein expression changes in HF ECM in response to mechanical stretch inhibited keratinocyte proliferation. It has previously been reported that the growth factor in ECM modulates ECM-mediated cell proliferation (23). To verify this in the present study, HFs seeded onto silicone membranes were cultured for six days under these three different stretch conditions and osmotically shocked. The remaining growth factors in ECM on silicone were eluted by high salt elution. Subsequently, HaCaT cells were seeded on silicone membrane containing growth factor-free ECM. The cyclic stretch-induced HF ECM on silicone membrane was still able to inhibit the proliferation of $\mathrm{HaCaT}$ cells (Fig. 3A).

Differential roles of collagen I and fibronectin in cell proliferation and migration. To further investigate the roles of different ECM components in cell proliferation, we prepared specific silicone gel membranes with single collagen I, fibronectin matrix or blank matrix. HaCaT proliferation analysis showed that membranes coated with collagen I or fibronection matrix promoted cell proliferation when compared with membrane without any ECM component coating (Fig. 3B). Furthermore, the collagen I-coated membrane had an even greater capability to promote cell proliferation compared with fibronectin-coated membrane (Fig. 3B). Data of cell cycle analysis was also consistent with this finding. We found that the percentage of G0/G1 cells in collagen I matrix group was significantly lower than that in fibronectin matrix and blank matrix groups (both $\mathrm{P}<0.01)$. In contrast, of the percentage of cells in $\mathrm{S}$ phase were markedly higher in collagen I matrix group compared with the other two groups (both $\mathrm{P}<0.01$; Fig. 3C). Next, we applied cell scratch experiment to analyze $\mathrm{HaCaT}$ migration in silicone gel membrane with different matrices. To exclude the effects of cell proliferation to cell migration, cells were treated for $48 \mathrm{~h}$ before scratch experiment with $10 \mu \mathrm{g} / \mathrm{ml}$ mitomycin for $1 \mathrm{~h}$. In the fibronectin matrix group, the healing area was 36.7 and $65.5 \%$ at 16 and $32 \mathrm{~h}$ after scratching (Fig. 3E). However, the healing area was quite lower in collagen I matrix group which was 17.8 and $37.2 \%$ at 16 and $32 \mathrm{~h}$ after scratching with representative images shown in Fig. 3E. In addition, since the high salt elution almost totally removed the growth factors in ECM, such an observation suggests that ECM-induced inhibition of cell proliferation under cyclic stretch was not a growth factors-related effect which was further proved by the western blot (Fig. 3D). Collectively, these results demonstrated that collagen I has a greater capability to promote cell proliferation while fibronectin has a higher capability to promote cell migration.

CASK suppression attenuates the effects of cyclic stretch-induced inhibition of HF proliferation. To investigate the underlying mechanism by which mechanical stretch inhibits cell proliferation, we performed an immunoprecipitation assay for integrin $\beta 1$, one of the most important integrin protein in ECM, and found that it bound with CASK (Fig. 4A). With mammalian two-hybrid analysis, we confirmed that CASK directly interacted with integrin $\beta 1$ (Fig. 4B). Using siRNA interference to suppress the expression of CASK in HFs under cyclic stretch, the results showed that CASK knockdown attenuated the inhibition of cell proliferation under cyclic stretch (Fig. 4C), indicating the important role of CASK in cyclic stretch related cell proliferation inhibition. Cell cycle analysis also indicated that the portion of G0/G1 cells in CASK knockdown HF cells was significantly lower compared with HF cells with normal CASK expression under cyclic stretch, while the percentage of HF cells in $\mathrm{S}$ phase was significantly higher (all $\mathrm{P}<0.01$; Fig. 4D). Western blot further demonstrated that the expression of collagen I increased and fibronectin decreased in CASK knockdown group compared with normal CASK expression HF cells (Fig. 4E). Collectively, these data implied that cyclic stretch-induced inhibition of $\mathrm{HF}$ proliferation could possibly links with the integrin $\beta 1$-CASK signal pathway, which might regulate the expression of specific protein expression in HF ECM.

\section{Discussion}

The capacity for tension endurance by skin tissues is crucial for skin development, maintenance of skin normal physiological functions and skin injure repair $(2,3)$. Using an in-house built cell mechanical stretch device, we found that the cyclic tension changed in skin tissues could be mimicked in vitro and the impact of cyclic tension change on proliferation and migration of $\mathrm{HF}$ and keratinocytes were further investigated in the current study. The results indicated that cyclic stretch significantly inhibited the proliferation of HF. Such inhibition of HF proliferation was associated with altered ECM protein levels in HF cells. We further showed that cultured keratinocytes on the altered of HF ECM inhibited the proliferation of keratinocytes, suggesting that the secreted ECM by HF under cyclic stretch might transmit the signal of cell proliferation inhibition. Data obtained from this study indicated a possible 'cross-talk' between HFs, ECM and keratinocytes in cutaneous tissues. Such interaction maybe involved in the processes underlying skin development, physiological skin function maintenance, skin injure repair and the formation of scars.

Although it is well known that multiple growth factors in ECM are able to regulate cell proliferation $(24,25)$, we demonstrated that growth factor-free ECM from cyclic stretched HF cells after removing the growth factors in ECM by high salt elution was able to induce inhibit the proliferation of keratinocytes. By analyzing the expression levels of major ECM components, we showed that the expression levels of collagen I and III, and fibronectin changed in response to cyclic stretch. We therefore concluded that not only the changes of growth factors in ECM, but also the changes of protein expression levels of ECM induced by skin tension alteration modulated the cell proliferation and migration in skin tissues.

Integrins are receptors coupling ECM components outside a cell to the cytoskeleton inside the cell, through the interaction with their ligands including collagen I and fibronectin (25). Integrins transmit the extracellular signals into the cells to in regulate multiple cellular processes in cell proliferation (26-29). Peripheral plasma membrane protein CASK could bind cell-surface proteins to coordinate signal transduction pathways (29). We showed in the present study that CASK was a partner of integrin $\beta 1$, and the integrin $\beta 1$-CASK pathway may be involved in the cyclic stretch-induced inhibition of HFs 
A

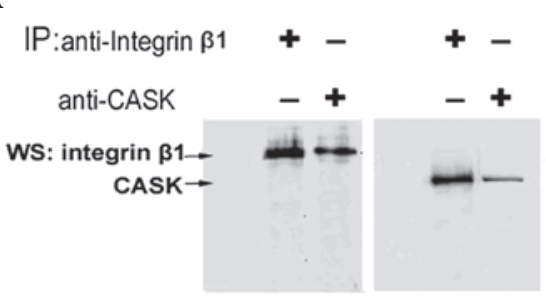

B

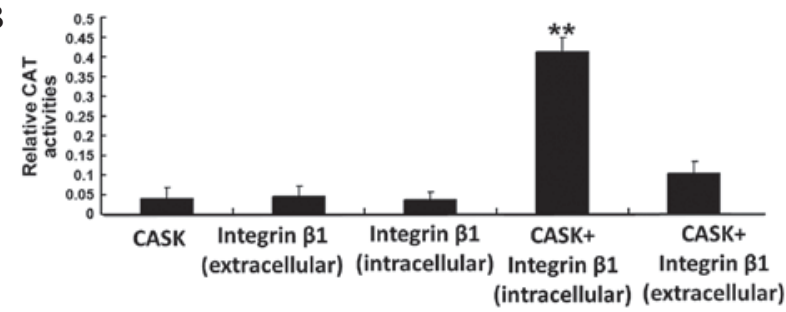

$\mathbf{C}$

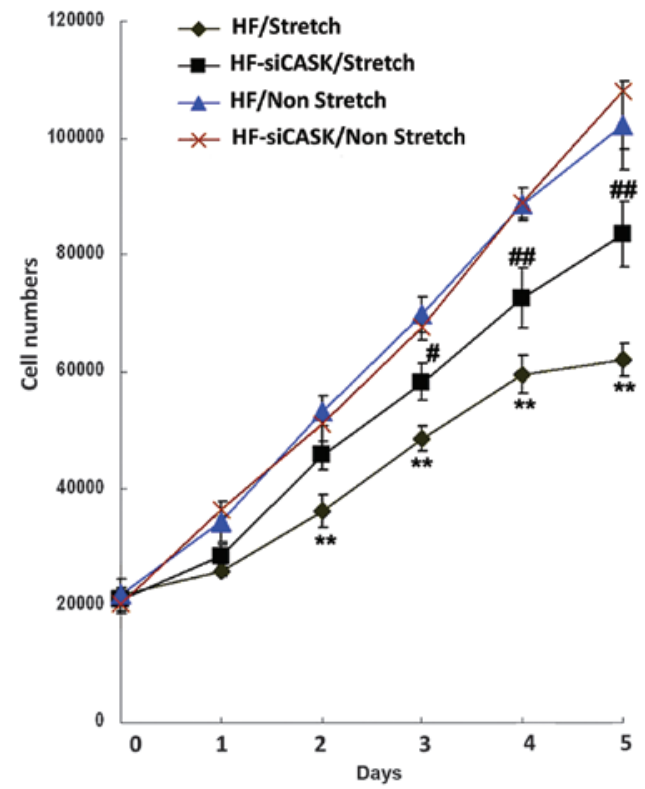

$\mathbf{F}$
D

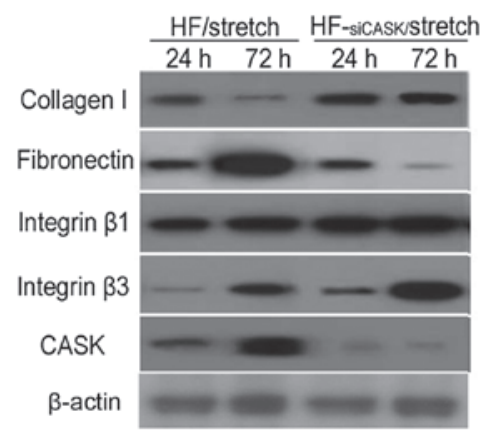

$\mathbf{E}$

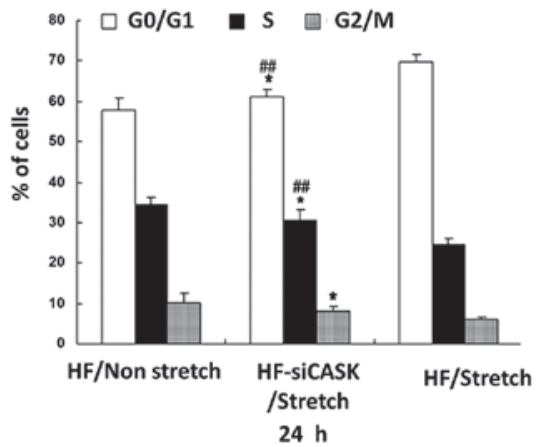

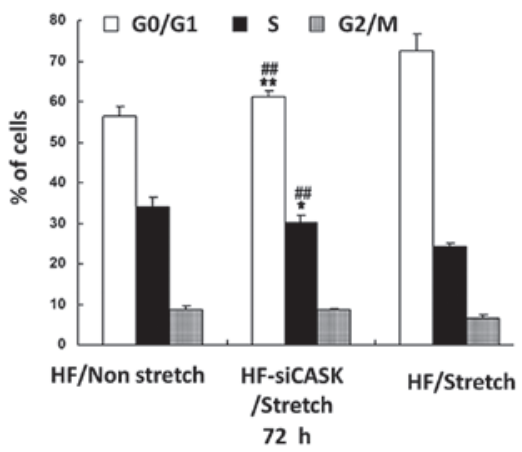

G

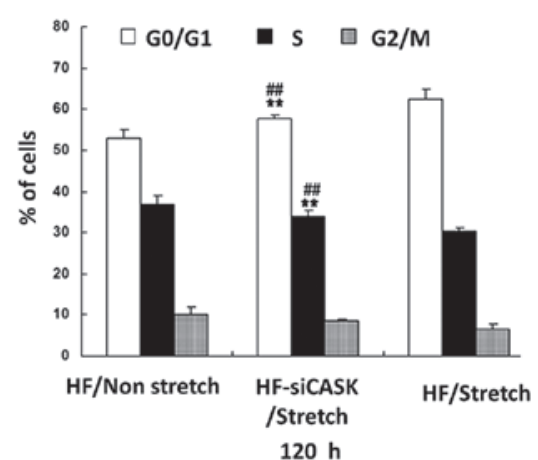

Figure 4. Integrin $\beta 1$-CASK signal pathway was involved in the HF proliferation of HF in response to cyclic mechanical stretch. (A) Coimmunoprecipitation of HFs cell lysates with CASK and integrin $\beta 1$. (B) Binding activities of CASK and integrin $\beta 1$ in HF cells $(n=6){ }^{* * *} \mathrm{P}<0.01 \mathrm{HF}$ cells co-transfected with pM-CASK and pVP16-integrin $\beta 1$ intracellular terminal vs. other groups. (C) Total HF cells with or without si-CASK knockdown under cyclic stretch, or non-stretch conditions for indicated periods of times. ${ }^{* *} \mathrm{P}<0.01 \mathrm{HF}$ cells/stretch vs. HF si-CASK cells/stretch; ${ }^{\# \#} \mathrm{P}<0.01 \mathrm{HF}$ si-CASK cells/stretch vs. HF cells/non stretch or HF si-CASK cells/non stretch. (D) Expression levels change of collagen I, fibronectin, integrin $\beta 1, \beta 3$, CASK of HF cells upon cyclic stretch with and without CASK knockdown. $\beta$-actin served as a loading control. Cell cycle analysis of HF cells with or without si-CASK knockdown cultured under cyclic stretch, or non-stretch for (E) 24, (F) 72 and (G) $120 \mathrm{~h}$. ${ }^{*} \mathrm{P}<0.05$ vs. static stretch or without stretch group; ${ }^{* *} \mathrm{P}<0.01$ si-CASK HF cells/stretch vs. HF cells/stretch; ${ }^{*} \mathrm{P}<0.05$ HF si-CASK cells/stretch vs. HF cells/non stretch or HF si-CASK cells/non stretch. ${ }^{\# \#} \mathrm{P}<0.01$ si-CASK HF cells/stretch vs. HF cells/non stretch HF, human skin fibroblast; CASK, calcium/calmodulin-dependent serine protein kinase.

proliferation. By knocking down the CASK expression with siRNA interference, we observed that the inhibition of HF proliferation was attenuated and the expression of collagen I increased while fibronectin decreased under cyclic stretch condition, suggesting that CASK was one of the upstream molecules in regulating the expression of ECM proteins in response to skin tension. Comprehensively, the present study indicated a possible molecular mechanism that skin tissues adapt cyclic stretch by regulation of the proliferation and migration of HFs and keratinocytes through integrin $\beta 1$-CASK signal pathway.

Since skin injury is associated with a sudden disappearance of local tension and would healing requires skin cell proliferation and migration, we further hypothesized that the 
local tension disappearance in injured skin might discharge the effects of proliferation inhibition, which subsequently initiate the process of wound healing. Previous studies have indicated that the disappearance of skin tension upregulated the expression of multiple proteinases in keratinocytes, which contributed to the cell proliferation and migration and promotes re-epithelization of skin (7-10). The present study further showed that the disappearance of skin tension could regulate the expression levels of major ECM components, which is consistent with a previous report indicating changes of mRNA levels of major ECM components in response to mechanical stretch (30). These results indicate the existence of other mechanisms by which the changes of skin tension could affect the biological functions of $\mathrm{HF}$ and keratinocytes by regulating the expression level of ECM components.

Although all biological functions of collagen I and III, and fibronectin in pathological and physiological states are not elaborately classified $(31,32)$, our results showed that different ECM components have differential biological functions, where collagen I and III might preferably regulate keratinocyte proliferation while fibronectin had a more dominant role in modulating keratinocyte migration. Since collagen and fibronectin are integrin ligands, the subtle functional differences between collagen and fibronectin in ECM implied the elaborate regulation of integrin $\beta 1-\mathrm{CASK}$ signal pathway in response to the changes of skin tension.

In conclusion, our results demonstrated the existence of HF-ECM-keratinocyte interaction in cutaneous tissues. The integrin $\beta 1-C A S K$ signal pathway in HFs may be involved in the outside-in signal transduction of extracellular stretch and altered the expression and secretion of ECM components in this process.

\section{Acknowledgements}

This study was supported by grants from the National High Technology Research and Development Program of China (grant no. 2011CB710904) and the National Natural Science Foundation of China (grant no. 81372813).

\section{References}

1. Silver FH, Siperko LM and Seehra GP: Mechanobiology of force transduction in dermal tissue. Skin Res Technol 9: 3-23, 2003.

2. Ingber DE: Mechanobiology and diseases of mechanotransduction. Ann Med 35: 564-77, 2003.

3. Ingber DE: Cellular mechanotransduction: Putting all the pieces together again. FASEB J 20: 811-27, 2006.

4. Bhadal N, Wall IB, Porter SR, Broad S, Lindahl GE, Whawell S and Lewis MP: The effect of mechanical strain on protease production by keratinocytes. Br J Dermatol 158: 396-398, 2008.

5. Jensen PJ and Lavker RM: Urokinase is a positive regulator of epidermal proliferation in vivo. J Invest Dermatol 112: 240-224, 1999.

6. McNeill $\mathrm{H}$ and Jensen PJ: A high-affinity receptor for urokinase plasminogen activator on human keratinocytes: Characterization and potential modulation during migration. Cell Regul 1: 843-852, 1990.

7. Wang XQ, Sun P and Paller AS: Gangliosides inhibit urokinase-type plasminogen activator (uPA)-dependent squamous carcinoma cell migration by preventing uPA receptor/alphabeta integrin/epidermal growth factor receptor interactions. J Invest Dermatol 124: 839-848, 2005.

8. Singer AJ and Clark RA: Cutaneous wound healing. N Engl J Med 341: 738-46, 1999.
9. Bissell MJ, Hall HG and Parry G: How does the extracellular matrix direct gene expression? J Theor Biol 99: 31-68, 1982.

10. Aszódi A, Legate KR, Nakchbandi I and Fässler R: What mouse mutants teach us about extracellular matrix function. Annu Rev Cell Dev Biol 22: 591-621, 2006.

11. Myllyharju J and Kivirikko KI: Collagens, modifying enzymes and their mutations in humans, flies and worms. Trends Genet 20: 33-43, 2004.

12. Carrion K, Dyo J, Patel V, Sasik R, Mohamed SA, Hardiman G and Nigam V: The long non-coding HOTAIR is modulated by cyclic stretch and WNT/ $\beta$-CATENIN in human aortic valve cells and is a novel repressor of calcification genes. PLoS One 9: e96577, 2014.

13. Zhu ZQ, Wang D, Xiang D, Yuan YX and Wang Y: Calcium/calmodulin-dependent serine protein kinase is involved in exendin-4-induced insulin secretion in INS-1 cells. Metabolism 63: 120-126, 2014.

14. Sun R, Su Y, Zhao X, Qi J, Luo X, Yang Z, Yao Y, Luo X and Xia Z: Human calcium/calmodulin-dependent serine protein kinase regulates the expression of $\mathrm{p} 21$ via the $\mathrm{E} 2 \mathrm{~A}$ transcription factor. Biochem J 419: 457-466, 2009.

15. Rodriguez-Mora OG, LaHair MM, McCubrey JA and Franklin RA: Calcium/calmodulin-dependent kinase I and calcium/calmodulin-dependent kinase kinase participate in the control of cell cycle progression in MCF-7 human breast cancer cells. Cancer Res 65: 5408-5416, 2005.

16. Soltow QA, Lira VA, Betters JL, Long JH, Sellman JE, Zeanah EH and Criswell DS: Nitric oxide regulates stretch-induced proliferation in $\mathrm{C}_{2} \mathrm{C}_{12}$ myoblasts. J Muscle Res Cell Motil 31: 215-225, 2010.

17. Eckes B, Nischt R and Krieg T: Cell-matrix interactions in dermal repair and scarring. Fibrogenesis Tissue Repair 3: 4-14, 2010.

18. Yin J, Ren W, Duan J, Wu L, Chen S, Li T, Yin Y and Wu G: Dietary arginine supplementation enhances intestinal expression of SLC7A7 and SLC7A1 and ameliorates growth depression in mycotoxin-challenged pigs. Amino Acids 46: 883-892, 2014

19. Yin J, Wu MM, Xiao H, Ren WK, Duan JL, Yang G, Li TJ and Yin YL: Development of an antioxidant system after early weaning in piglets. J Anim Sci 92: 612-619, 2014.

20. Chen L, Qiu J, Yang C, Yang X, Chen X, Jiang J and Luo X: Identification of a novel estrogen receptor betal binding partner, inhibitor of differentiation-1, and role of ERbeta1 in human breast cancer cells. Cancer Lett 278: 210-219, 2009.

21. Qi J, Su Y, Sun R, Zhang F, Luo X, Yang Z and Luo X: CASK inhibits ECV304 cell growth and interacts with Id1. Biochem Biophys Res Commun 328: 517-521, 2005.

22. Kurita M, Okazaki M, Fujino T, Takushima A and Harii K: Cyclic stretch induces upregulation of endothelin-1 with keratinocytes in vitro: Possible role in mechanical stress-induced hyperpigmentation. Biochem Biophys Res Commun 409: 103-107, 2011.

23. Fuentes-Calvo I, Blázquez-Medela AM, Eleno N, Santos E, López-Novoa JM and Martínez-Salgado C: H-Ras isoform modulates extracellular matrix synthesis, proliferation and migration in fibroblasts. Am J Physiol Cell Physiol 302: C686-C697, 2012.

24. Singh P, Chen C, Pal-Ghosh S, Stepp MA, Sheppard D and Van De Water L: Loss of integrin alpha9beta1 results in defects in proliferation, causing poor re-epithelialization during cutaneous wound healing. J Invest Dermatol 129: 217-228, 2009.

25. Bush KA and Pins GD: Carbodiimide conjugation of fibronectin on collagen basal lamina analogs enhances cellular binding domains and epithelialization. Tissue Eng Part A 16: 829-838, 2010

26. Araki E, Momota Y, Togo T, Tanioka M, Hozumi K, Nomizu M, Miyachi Y and Utani A: Clustering of syndecan-4 and integrin betal by laminin alpha 3 chain-derived peptide promotes keratinocyte migration. Mol Biol Cell 20: 3012-3024, 2009.

27. Mukoyama Y, Utani A, Matsui S, Zhou S, Miyachi Y and Matsuyoshi N: T-cadherin enhances cell-matrix adhesiveness by regulating betal integrin trafficking in cutaneous squamous carcinoma cells. Genes Cells 12: 787-796, 2007.

28. Rodius S, Indra G, Thibault C, Pfister V, Georges-Labouesse E: Loss of alpha6 integrins in keratinocytes leads to an increase in TGFbeta and AP1 signaling and in expression of differentiation genes. J Cell Physiol 212: 439-449, 2007. 
29. Hsueh YP, Wang TF, Yang FC and Sheng M: Nuclear translocation and transcription regulation by the membrane-associated guanylate kinase CASK/LIN-2. Nature 404: 298-302, 2000.

30. Sawaguchi N, Majima T, Funakoshi T, Shimode K, Harada K, Minami A and Nishimura S: Effect of cyclic three-dimensional strain on cell proliferation and collagen synthesis of fibroblast-seeded chitosan-hyaluronan hybrid polymer fiber. J Orthop Sci 15: 569-77, 2010.
31. Teti A: Regulation of cellular functions by extracellular matrix. J Am Soc Nephrol 2 (Suppl): S83-S87, 1992.

32. Wang H, Yan X, Shen L, Li S, Wang S, Hou XL, Shi C, Yang Y, Dai $J$ and Tan Q: Acceleration of wound healing in acute full-thickness skin wounds using a collagen-binding peptide with an affinity for MSCs. Burn Trauma 2: 181-186, 2014. 